\title{
ANGLICISMOS EN EL ESPAÑOL DE AMÉRICA
}

\author{
GÜNTHER HAENSCH \\ Universidad de Augsburgo
}

\section{Resumen}

El propósito de este artículo consiste en presentar una pequeña lista de anglicismos de uso frecuente en el español de América. La información acerca de estas unidades, que ha sido obtenida a través de informants natives y de Fuentes lexicográficas, puede arrojar nueva luz acerca de las diferencias entre el español europeo y el español americano.

PALABRAS CLAVE: préstamo léxico, anglicismo, español de América, lexicografia, dialectología.

\begin{abstract}
The aim of this paper is to present a short list of anglicisms which are frequently used in American Spanish. The information about these units, obtained by native informants and lexicographical sources, may shed new light on the differences between European and American Spanish.
\end{abstract}

KEY WORDS: loanword, anglicism, American Spanish, lexicography, dialectology.

\section{Introducción}

El objetivo de este artículo es ofrecer una selección de anglicismos de uso en el español corriente de los países hispanoamericanos, para ver en qué se distingue el uso de anglicismos en el español de América del uso del español de España. Los materiales léxicos aprovechados para este trabajo proceden, en su mayor parte, de una encuesta que realicé, hace ya unos años, mediante la consulta de informantes, todos ellos lingüistas, en los 19 países hispanoamericanos, quienes contestaron un cuestionario sobre 750 unidades léxicas de las que se podía suponer que podrían presentar diferencias frente al español peninsular. Otros materiales léxicos proceden de tres de los diccionarios del español de América elaborados en la Universidad de Augsburgo: el Nuevo Diccionario de Colombianismos (NDC), el Diccionario del Español de Cuba-Español de España y el Diccionario del Español de Argentina-Español de España (ver los datos bibliográficos en la lista de abreviaturas más abajo).

No se han tenido en cuenta los numerosos anglicismos que tienen el mismo significado y las mismas condiciones de uso en España e Hispanoamérica, pero sí aquéllos que presentan alguna diferencia entre ambas áreas, como pueden ser: significado diferente, diferencias formales, cambio de la categoría gramatical y frecuencia de uso.

En cuanto a los anglicismos del español de América, se indica, mediante marcas diatópicas, en qué país o países de Hispanoamérica se usan. Estas indicaciones no pretenden ser exhaustivas. Cuando señalo, por ejemplo, que una palabra se usa en Cuba, esto no quiere decir que no se use también en otros países hispanoamericanos para los que, de momento, carezco de documentación. Contrariamente a la práctica de muchos diccionarios monolingües y bilingües, que ponen a cualquier palabra usual en un solo país americano $-o$ en 
dos o tres de ellos - la marca $A m$ que hace pensar que ésta se usa en toda Hispanoamérica, quiero ofrecer datos seguros sobre el uso de los anglicismos en los distintos países. El hecho de que se registre un anglicismo con un determinado significado no implica que no haya también sinónimos del mismo formados con elementos españoles. Por ejemplo, junto al anglicismo concreto ('hormigón') se usa en una serie de países hispanoamericanos también la voz hormigón.

\section{Abreviaturas y símbolos}

En nuestro glosario de anglicismos del español de América se usan las siguientes abreviaturas:

\subsection{Marcas diatópicas}

$\begin{array}{llll}\text { Am } & \text { América } & \text { Méx } & \text { México } \\ \text { Arg } & \text { Argentina } & \text { Nic } & \text { Nicaragua } \\ \text { Bol } & \text { Bolivia } & \text { Pan } & \text { Panamá } \\ \text { Chil } & \text { Chile } & \text { Par } & \text { Paraguay } \\ \text { Col } & \text { Colombia } & \text { Pe } & \text { Perú } \\ \text { Cu } & \text { Cuba } & P R & \text { Puerto Rico } \\ E c & \text { Ecuador } & R D & \text { República Dominicana } \\ \text { Esp } & \text { España } & \text { Salv } & \text { El Salvador } \\ G B & \text { inglés británico } & U r & \text { Uruguay } \\ \text { Guat } & \text { Guatemala } & U S & \text { inglés americano } \\ \text { Hond } & \text { Honduras } & \text { Ven } & \text { Venezuela }\end{array}$

\subsection{Abreviaturas bibliográficas}

DEA Manuel Seco et al.: Diccionario del español actual. Aguilar. Madrid 1999.

DEArg Günther Haensch y Reinhold Werner (coords.): Diccionario del Español de Argentina-Español de España. Gredos. Madrid 2000.

$D E C u \quad$ Gisela Cárdenas, Antonia $\mathrm{M}^{\mathrm{a}}$ Tristá y Reinhold Werner (coords.): Diccionario del Español de Cuba-Español de España. Gredos. Madrid 2000.

NDA Félix Rodríguez y Antonio Lillo: Nuevo diccionario de anglicismos. Gredos. Madrid 1997.

NDC Günther Haensch y Reinhold Werner (coords.): Nuevo Diccionario de Colombianismos. Instituto Caro y Cuervo. Bogotá 1997.

\subsection{Otras abreviaturas}

adj adjetivo

adv adverbio

$f \quad$ sustantivo femenino

id. La voz inglesa tiene el mismo significado que el americanismo que procede de ella 
ingl. inglés.

inv invariable.

$m$ sustantivo masculino

pl plural

var: variante

$v t \quad$ verbo transitivo

$<\quad$ procede de

\section{Glosario de una selección de anglicismos del español de América}

all around [olarráun] 1 adj $\mathrm{Cu}$ '(persona) capaz de trabajar en diferentes oficios'; $2 \mathrm{~m} \mathrm{Cu}$ 'joven que hace trabajos menores y recados', 'botones' $<$ ingl. all around adj '(persona) con capacidad para todo'.

apartamento $m$ Col, $C R, C u$, Guat, Hond, Nic, Pan, Par, PR, RD, Salv, Ven 'unidad de vivienda de cualquier extensión', Esp: piso. En $E s p$, ‘vivienda en una casa moderna de pisos, normalmente con solo dos o tres habitaciones' ( $D E A)$; cuando es más pequeña, en Esp: estudio $m<$ ingl. apartment 'piso', 'vivienda'.

balancear $v t$ general en $A m$ 'equilibrar (las ruedas de un automóvil)' < ingl. to balance, $i d$.

básquetbol $m$ var. basquetbol general en $A m<i n g l$. basketball, $i d$. Esp: baloncesto. Se conoce y se usa básquetbol en Esp, pero es más frecuente baloncesto.

bife $m$ Arg, Par, Ur 'trozo de carne vacuna para asar', 'filete' < ingl. beefsteak, id. Según el $D E A$, en $E s p$ la voz bife sirve para designar el filete al estilo argentino.

bloomer $m C R$, Cu, Hond, Nic, PR, Salv; blúmers mpl Ven 'braga o eslip de mujer' <ingl. bloomer (hoy arcaico), id. La etimología de esta voz es curiosa.En EE.UU., Mrs. Amelia Bloomer propuso en el siglo XIX una ropa adecuada para la mujer, que consistía en una camisa corta y pantalón largo. Más tarde pasó a significar sólo 'pantalón' y finalmente 'braga'. En ingl. actual: knickers, pants.

bluyín $m$ Col 'vaqueros', 'tejanos'; también: yin $m$ Ur; bluyíns Chi, Ven; jeans Méx; jean Arg < ingl. blue jeans, id. La pronunciación es siempre [yin] o [yins].

brassiere var: brasier $m$ Col, Méx, Ven 'sujetador', 'sostén' < ingl. brassiere, id. Frecuentemente truncado: bra.

bróder $m$ Col, Ven. Voz coloquial para dirigirse a amigos, compañeros de trabajo y, en general, a personas de la misma clase social, como en Esp compa, y usada entre jóvenes como tratamiento, como en Esp tío, macho < ingl. brother 'hermano'. 
carro $m$ Centroamérica, Caribe, Col, Ven, Ec, 'automóvil', 'turismo'; en Esp coche < ingl. car, id. En el Cono Sur se usa auto $m$.

chequear $v t$ Am significa como en $E s p$ 'someter a revisión o control'; en Am significa además 'facturar el equipaje en un aeropuerto' < ingl. to check in, $i d$.

chica de tapa $f$ Méx 'mujer bonita cuya foto aparece en la portada de revistas' < ingl. cover girl, id.

cloche $m C u, P R, R D$, Ven; cloch $m$ Col, $C R$, Guat, Hond, Méx, Pan 'embrague de un automóvil' < ingl. clutch, id.

clóset $m$ Bol, Chi, Col, CR, Cu, Ec, Guat, Hond, Méx, Nic, Pan, Pe, RD, Salv, Ven 'armario empotrado' < ingl. Closet 'armario'. En Arg y $U r$ se usa el galicismo placar(d) con el mismo significado.

cocoa $f 1 C u$ 'polvo fino y amargo de cacao' $(D E C u)$; 2 Col 'chocolate en polvo de preparación instantánea' $(N D C)<$ ingl. cocoa 'cacao', 'chocolate'.

coctel $m$ 'bebida preparada por la mezcla de varios licores y otros ingredientes' $(D E A)$. Se usa con otra acentuación que en Esp, donde la forma más usual es cóctel < ingl. cocktail, id. En Arg significa además: 'huevo batido con vino'.

concreto $m$ En $A m$ 'hormigón' < ingl. concrete, $i d$. En muchos países hispanoamericanos se usa, junto a este anglicismo, la palabra hormigón.

condominio $m$ Chi, CR, Guat, Hond, Méx, Pan, PR, RD, Salv, Ven "vivienda en propiedad horizontal' $<$ ingl. condominium, $i d$.

Corte $f$ general en $A m$ 'tribunal de justicia' < ingl. court, $i d$.

Crack $m$ Arg, $U r$ 'persona que destaca en una actividad', 'hacha', 'as' < ingl. crack, id.

cuáqueres, var. cuáquers $m p l$ Arg 'copos de avena' < ingl. Quakers 'miembros de la secta religiosa de los cuáqueros o Sociedad de Amigos'.Originariamente, nombre de una marca comercial de copos de avena. En ingl., 'copos de avena' = oat meal, rolled oats.

curita $f$ general en $A m$ 'tira de vendaje plástico' < ingl. curity, nombre de marca comercial norteamericana, lo mismo que su equivalente en Esp: tirita.

deforestación $f C r, R D$ 'tala de árboles', 'desmonte' < ingl. deforestation, id.

direccional $f C o l$, Méx, Pan, $P e, R D$ 'intermitente de un automóvil para indicar un cambio de dirección' < ingl. directional light, $i d$. 
directorio (telefónico) $m \mathrm{Col}, C R, M e ́ x$, Pan, $R D$, Salv 'guia telefónica'<ingl. (telephone) directory, $i d$.

durmiente $m$ Arg, Bol, Chi, Col, CR, Guat, Hond, Méx, Nic, Pan, RD, Salv 'traviesa (en la vía del tren)' $<G B$ sleeper ( $U S$ : track).

elevador $m C u$, Méx 'ascensor (para personas)' < US elevator, id. En Esp, elevador designa un 'aparato o dispositivo para elevar, especialmente materiales a granel o cargas' $(D E A)$.

Estación de policía $f C o l, C R, C u$, Guat, $R D$ 'oficina o puesto de policía en una determinada demarcación urbana'. Esp comisaría $<$ ingl. police station, $i d$.

fólder $m \mathrm{Bol}$, Col, $\mathrm{CR}, \mathrm{Cu}, \mathrm{Ec}$, Hond, Méx, Nic, Pan, Pe, PR, RD, Salv, Ur 'carpeta (para guardar papeles)' < ingl. folder, id.

freezer [friser], var. friser $m \mathrm{Arg}, \mathrm{Col}$ 'congelador' < ingl. freezer, id.

full adj inv Ven 'lleno (referido especialmente al depósito de gasolina)' < ingl. full, 'lleno'. A todo ful(l) $a d v \mathrm{Col}, \mathrm{Cu}$, $\mathrm{Ven}$ 'deprisa', 'a toda pastilla', 'intensamente'.

gasfiter $m C h i$, gasfitero $m P e$ 'fontanero (especializado en la instalación del gas)' < ingl. gasfitter, $i d$.

guachimán $m$ Chi, Col, $C R, N i c, P e, P R$ (obsoleto), Ven 'vigilante' < ingl. watchman, id.

jogging $m$ significa en $E s p$ como en $A m$ 'ejercicio físico que consiste en correr a poca velocidad durante cierto tiempo para mantenerse en forma'. En Arg significa además 'conjunto deportivo de chaqueta o sudadera y pantalón largo a juego', Esp chándal < ingl. jogging, id.

kindergarten, generalmente truncado en kinder $m \mathrm{Arg}, \mathrm{Chi}, \mathrm{Col}, \mathrm{CR}$, Guat, Hond, Méx, Nic, Pan, Par, Pe, RD, Salv, Ven 'parvulario', 'jardín de infancia'. La palabra es de origen alemán. El primer kindergarten fue fundado por el pedagogo alemán Friedrich Fröbel en 1837 y lleva este nombre desde 1840. La palabra vino a través de los EE.UU. Por eso se encuentra en $A m$ a veces la grafía kindergarden, con la - $d$ - del ingl. garden, 'jardín'.

kitchenette $f A r g, C h i, P e, U r, V e n$ 'rincón-cocina'. Según el $D E A$ y el $N D A$, se usa en $E s p$ con el mismo significado. Parece de uso más frecuente en $A m$.

licencia de conducir $A r g$ y muchos otros paises de Am 'permiso de conducir'; calco del ingl. driver's licence, $i d$.

llamada de larga distancia general en $\mathrm{Am}$ 'llamada telefónica interurbana o internacional'. Esp 'conferencia'; calco del inglés long distance call, $i d$. 
Iunch $m C u$, lonch $m P R$, lonche $m C o l$ 'plato ligero que se toma entre las comidas' < ingl. lunch 'comida del mediodía'. Derivados en Col: lonchería $f$ 'cafetería' y lonchera $f$ 'fiambrera'.

mitín $m$ Arg 'reunión de carácter político o social', en Esp con otra acentuación: mítin < ingl. meeting, id.

overol $m$ general en $A m$ 'mono de trabajo con pantalón de peto' < ingl. overalls, id.

pantry $m C u$ 'despensa' < ingl. pantry, id.

Panty $m$ Bol, Chi, CR, Cu, Guat, Hond, Nic, Pe, Salv, Ven pantimedias fpl Méx "media-pantalón' $<U S$ pantyhose ( $G B$ tights).

picles $\mathrm{mpl} \mathrm{Am}$ 'verduras conservadas en un preparado con vinagre y sal', Esp encurtidos $<$ ingl. pickles, $i d$.

pick-up $m$, var. picó $m$ Col, $C u$ 'camioneta con una plataforma de carga' < ingl. ick up (truck), $i d$.

pico: hora(s) pico $\mathrm{Arg}, \mathrm{Col}, \mathrm{CR}, \mathrm{Cu}$, Guat, Méx, Nic, Par, RD, Salv, Ur, Ven, hora pic Chi 'hora(s) punta (de mayor afluencia de tráfico)' < ingl. pick hours, id.

piyama $m$ Arg, Bol, Par, Ur; piyama $f C o l, C R, C u$, Guat, Méx, Nic, Par, RD, Salv, Ur, Ven; payama $C u$, payamas $P R$. En Esp: pijama $m$. La palabra es de origen indio (de la lengua urdu) y significaba primero 'pantalones bombachas' (como los llevan los musulmanes de ambos sexos). Luego designaba un traje de tela muy ligera (seda o algodón) que se llevaba en la India bajo dominación inglesa. Los ingleses introdujeron la palabra en su país con el significado actual de 'traje de dormir compuesto de chaqueta y pantalón' $(D E A)$, primero en la forma pajamas, sustituida a finales del siglo XIX por pyjamas. En US se usa payamas. Las formas con $-i$ - parecen proceder del inglés británico, las de Cuba y Puerto Rico, con $-a^{-}$, del inglés americano.

pluma fuente $f \mathrm{Arg}, \mathrm{Bol}, \mathrm{Chi}, \mathrm{CR}, \mathrm{Cu}$, Guat, Méx, Nic, Pan, Pe, PR, Salv, Ven 'estilográfica'; calco del ingl. fountain pen, $i d$.

policía acostado $m \mathrm{Col}$ (y otros países) 'en las calles y carreteras de zonas urbanas, ligera elevación del pavimento que se extiende de una orilla a otra del mismo para obligar a los automovilistas a aminorar la marcha' (NDC). Los policías acostados son más altos que las bandas sonoras. Según me informaron taxistas de Madrid y Alicante, los policías acostados se llaman en su jerga profesional guardias dormidos o guardias muertos.

ranquear $v t C u$ 'asignar a una persona o a un club deportivo un lugar determinado en una clasificación' < ingl. to rank, id. En Esp se usa el sustantivo ranking (NDA). 
receso $m$ Arg, Col, Cu, Guat, Méx, Nic, $P R$, Pe, Salv 'suspensión de las actividades de administraciones públicas, tribunales, etc.' < ingl. recess, $i d$. Según el $D E A$, la voz es en $E s p$ de estilo elevado o humorístico y parece tener un índice de frecuencia más bajo que en América.

récord $m C u$ 'registro de datos profesionales de una persona' < ingl. record 'registro', 'documento'.

reforestar $v t$ general en $A m$ 'repoblar un terreno con árboles' < US reforest, id. (GB: reafforest).

reforestación $f$ general en $A m$ 'repoblación forestal' < ingl. reforestation, id. En Esp empieza a usarse en la terminología forestal.

reportar $v t$ Am 'informar'; reportarse 'personarse o dar noticias (una persona ausente; por ej., por teléfono)' < ingl. to report 'informar, relatar'.

reversa $f$ Arg, Col, $C R, M e ́ x, P R, R D, U r$; reverso $m$ Col, Hond 'marcha atrás en el automóvil' < ingl. reverse gear, $i d$.

scotch (tape) [eskótchtei] $m$ Arg, Bol, Chi, Cu, Ec, Hond, Méx, Par, Pe, Salv, Ur "cinta adhesiva'. Es nombre de marca lo mismo que su equivalente peninsular cel.lo $\mathrm{m}$. En $\mathrm{Am}$ es de uso más frecuente la forma abreviada Scotch.

shopping $m \mathrm{Cu}$ 'tienda en la que se pueden comprar artículos sólo con divisas, generalmente dólares' $(D E C u)<$ ingl. shopping centre.

sleeping (bag) $m$ Bol, Col, CR, Guat, Méx, Pan, Pe, Ven 'saco de dormir' < ingl. sleeping bag, $i d$.

spike [espái] $m \mathrm{Cu}$ 'zapatilla deportiva con clavos' < ingl. spikes, $i d$.

steward $f C R$; estiguar $f$ Guat; stúar $f$ Salv 'azafata' < ingl. steward, cuya forma femenina es stewardess, $i d$.

straight [estrái] adj $\mathrm{Cu}$ '(referido al trago de una bebida alcohólica) pura, de alta graduación' < ingl. straight, id.

straple [estráple] $m C u 1$ 'blusa o vestido de mujer sin tirantes'; 2 'sujetador sin tirantes' $<$ ingl. strapless adj 'sin tirantes'.

suspensores $m p l$ Arg 'tirantes' < US suspenders, id. (GB: braces).

switch $m C u$, Guat, Hond, Pe, PR, RD, Salv; suiche $m$ Col, CR, Pan, Ven 'interruptor' < ingl. Switch, id. 
tanque $m$ Arg, Bol, Col, CR, Cu, Guat, Hond, Méx, Nic, Pan, Pe, Ur, Ven 'depósito de gasolina (de un automóvil)' $<$ ingl. tank, id.

tanquear $v t / i \mathrm{Col}$ 'echar gasolina' < ingl. to tank, id.

tanquero $m \mathrm{Cu}$ 'buque-cisterna' < ingl. tanker, $i d$.

toalla sanitaria $f$ 'apósito higiénico', 'compresa higiénica'; frecuente en muchos países hispanoamericanos como calco del ingl. sanitary towel, $i d$.

zipper, var. cíper $m C R, C u$, Guat, Méx, Nic, Pan, PR, RD, Salv 'cierre de cremallera' < ingl. zipper, id.

\section{Comentarios}

La mayoría de las voces de nuestro glosario son préstamos, a veces con cambios de significado, pero siempre con adaptación a la fonética del español de América. En los países que tienen o han tenido menos contactos con EE.UU. se tiende más a una adaptación gráfica y morfológica al sistema español que en los países con fuerte influencia norteamericana (México, Caribe, Centroamérica y, en parte, también Chile). Por ej.: lunch en Cuba, pero lonche en Colombia; gásfiter en Chile, pero gasfitero en Perú.

A pesar del rechazo del anglicismo por el régimen cubano actual, el número relativamente alto de anglicismos en el español actual de Cuba llama la atención. Es de suponer que éstos fueron introducidos antes de la Revolución y se han mantenido sin ser sustituidos por palabras formadas con elementos españoles. El Diccionario del Español de Cuba-Español de España, publicado en 2000, registra más anglicismos que no figuran en nuestro glosario.

Algunos de los anglicismos se usan en una forma elíptica o truncada. Por ej.: kinder por kindergarten, scotch por scotch tape, sleeping por sleeping bag, shopping por shopping centre, direccional < directional light. Ciertos adjetivos ingleses son sustantivados en español. Por ej.: strapless y allaround.

Los calcos por traducción literal de un modelo inglés son una posibilidad de adaptar el anglicismo fonéticamente y morfológicamente al sistema español: balancear, directorio, durmiente, estación de policía, llamada de larga distancia, pluma fuente, etc.

En unos países un anglicismo se usa con significado diferente de otros, en otros se usan en vez del anglicismo, o junto al anglicismo, palabras formadas con elementos españoles. Por ejemplo, en vez de guachimán se usan vigilante o celador; en vez de sleeping, o junto con esta voz, se usa bolsa de dormir (Ar, Bol, Pe, Ur), saco de dormir (Chi, Méx, Nic, Par, $\mathrm{RD}$, Ven), talego de dormir (Col); junto a bloomer o en vez de esta palabra se usa calzón (Bol, Chi, CR, Guat, Hond, Nic, Salv), pantaleta (Méx), etc.; en vez de gásfiter o gasfitero se usa en la mayoría de los países hispanoamericanos la palabra plomero.

En el español de América son bastante frecuentes los nombres de marca usados abusivamente como nombres comunes. En nuestro glosario ya hemos visto algunos ejemplos como cuáqueres en vez de copos de avena, scotch en vez de cinta adhesiva (Esp) o cinta pegante (Am). Otros ejemplos del español de América son: en el Cono Sur, gillette por hoja de 
afeitar, en Centroamérica, kótex por apósito higiénico o compresa higiénica; en Colombia se usa betamax en lugar degrabador de video o magnetoscopio.

\section{Conclusión}

Los préstamos y calcos léxicos procedentes del inglés son un factor importante de diferenciación del léxico de los distintos países hispanoamericanos, tanto frente al español peninsular como entre las variedades americanas del español; especialmente en aquellos casos en que la única palabra usual para designar un referente es un anglicismo, como en el caso de cloch(e) 'embrague del automóvil'. La distribución geográfica de los anglicismos y de su significado, o sus distintas acepciones en España e Hispanoamérica, parece arbitraria. Por lo tanto, el anglicismo tiene su propia geografía lingüística en el amplio mundo hispánico. 